\title{
Filigrane
}

Écoutes psychothérapiques

\section{Réflexions autour de l'accompagnement haptonomique de la grossesse}

\section{Florence Vinit}

Volume 18, numéro 2, automne 2009

Le corps. Sur le divan. Dans le fauteuil II

URI : https://id.erudit.org/iderudit/039288ar

DOI : https://doi.org/10.7202/039288ar

Aller au sommaire du numéro

\section{Éditeur(s)}

Revue Santé mentale au Québec

ISSN

1192-1412 (imprimé)

1911-4656 (numérique)

Découvrir la revue

\section{Citer cet article}

Vinit, F. (2009). Réflexions autour de l'accompagnement haptonomique de la grossesse. Filigrane, 18(2), 38-50. https://doi.org/10.7202/039288ar
Résumé de l'article

La réflexion psychanalytique, à travers notamment les travaux de Didier Anzieu et d'E. Bick a montré la place des échanges tactiles dans la structuration psychique de l'individu et ce qu'ils peuvent induire comme confirmation affective chez celui-ci. Dans le domaine de la périnatalité, l'haptonomie (F. Veldman) propose d'entrer en contact avec le bébé in utero à travers la qualité de présence de la main posée sur la paroi du ventre de la mère, le mouvement du bébé initiant en retour un dialogue avec le couple parental. Si l'haptonomie cherche avant tout à développer une interaction avec l'enfant à naître, elle ouvre un espace d'expression et d'accompagnement des problématiques d'attachement vécues par la mère suite à une fausse couche antérieure ou lors d'une grossesse à risque. Cet article exposera les présupposés sur lesquels se fonde l'haptonomie (notamment au niveau d'une phénoménologie du corps et de l'affectivité) et illustrera son propos de trois cas cliniques tirés de la pratique personnelle de l'auteur. 


\title{
Réflexions autour de I'accompagnement haptonomique de la grossesse
}

\author{
florence vinit
}

La réflexion psychanalytique, à travers notamment les travaux de Didier Anzieu et d'E. Bick a montré la place des échanges tactiles dans la structuration psychique de l'individu et ce qu'ils peuvent induire comme confirmation affective chez celui-ci. Dans le domaine de la périnatalité, l'haptonomie (F. Veldman) propose d'entrer en contact avec le bébé in utero à travers la qualité de présence de la main posée sur la paroi du ventre de la mère, le mouvement du bébé initiant en retour un dialogue avec le couple parental. Si l'haptonomie cherche avant tout à développer une interaction avec l'enfant à naître, elle ouvre un espace d'expression et d'accompagnement des problématiques d'attachement vécues par la mère suite à une fausse couche antérieure ou lors d'une grossesse à risque. Cet article exposera les présupposés sur lesquels se fonde l'haptonomie (notamment au niveau d'une phénoménologie du corps et de l'affectivité) et illustrera son propos de trois cas cliniques tirés de la pratique personnelle de l'auteur.

Psychoanalytic thinkers such as Didier Anzieu and Esther Bick have underlined the importance of touch in the psychic structuration of the subject and its impact on affective confirmation. In the prenatal field, haptonomy (F. Veldman) is a proponent of contact with the baby in utero through hand movements applied on the mother's belly and particular attention given to the foetus reaction as fostering a dialogue with the parental couple. Beyond facilitating interactions with the unborn child, haptonomy allows the expression and working through of attachment issues in mothers having undergone a previous stillbirth or facing a complicated pregnancy. This paper outlines the founding principles of haptonomy (mainly through corporal phenomenology and affectivity) and presents three clinical vignettes as illustrations.

\section{Introduction}

Dans le centre de la mère, la vie était d'une richesse infinie.

Sans parler des sons et des bruits, tout était, pour l'enfant,

Constamment en mouvement.

Que la mère vienne à se lever et marcher,

Qu'elle se tourne, qu'elle se baisse,

Ou qu'elle se hausse sur la pointe des pieds,

Qu'elle épluche les légumes ou manie le balai,

Autant de vagues,

Autant de sensations pour l'enfant.

Et que, même, la mère vienne à se reposer,

Qu'elle prenne un livre et s'asseye

Ou qu'elle se couche et s'endorme, 
Sa respiration n'en continue pas moins

Dans la houle paisible,

Le ressac,

Continue de bercer le bébé

(Leboyer, 1977, 13)

La réflexion psychanalytique, notamment à travers les travaux de Winnicott, de Didier Anzieu et d'Esther Bick a montré la place des échanges tactiles dans la structuration psychique de l'enfant et dans le processus d'attachement avec ses parents. Plus largement, un contact signifiant, qu'il passe ou non par une proximité physique, s'inscrit dans une confirmation affective propre à l'être humain (Buber, 1992). Dans le domaine de la périnatalité, l'approche haptonomique (Veldman, 1989), pratiquée en Europe depuis une vingtaine d'années, propose d'entrer en contact avec le bébé in utero à travers la qualité de présence de la main posée sur la paroi du ventre de la mère, le mouvement du bébé initiant en retour un dialogue avec le couple parental. Si l'haptonomie cherche avant tout à développer une interaction avec l'enfant à naître, elle ouvre un espace d'expression et d'accompagnement de certaines problématiques d'attachement entre la mère et l'enfant tout en interrogeant la place donnée à la sécurisation affective dans notre culture. Cet article exposera l'importance de la peau et du toucher dans le développement psychique, présentera les présupposés sur lesquels se fonde l'haptonomie avant d'illustrer son propos par des exemples cliniques.

\section{La peau et le toucher dans la structuration du psychisme:}

La conscience et le développement de la sensibilité ne sont pas spécialement encouragés dans notre société contemporaine, parfois même confondus avec une quête de sensations intenses, tel qu'en témoigne dans le social, le succès des sports extrêmes ou même celui des inscriptions et entailles corporelles: Le Breton (2002a, 2002b) souligne combien le besoin d'enracinement dans le corps peut passer, chez les jeunes gens d'aujourd'hui, par une prise de risque systématique ou par une impression d'exister recherchée à travers la douleur.

Dans la sphère professionnelle, la reconnaissance de la sensibilité ne fait pas plus l'objet d'une attention propre. La formation en science médicale et infirmière par exemple comprend l'acquisition d'un savoir théorique et pratique sans qu'une réflexivité sur le geste même du soin et sa réalisation n'ait réellement lieu (Vinit, 2007). Parmi certaines professions ayant fait du développement du sentir le cœur de leur pratique, la massothérapie et l'ostéopathie ont poussé très loin la capacité de la main à toucher les structures tissulaires du corps. Pour autant, elles n'ont pas construit une phénoménologie du toucher (ni du contact) équivalente sur le plan théorique à la sensibilité mise en œuvre (Courtecuisse, 2009).

Ces différents manques d'intérêt pour une théorisation de l'acte du toucher s'expliquent en partie par une tradition occidentale marquée par un long historique de mépris du corps et notamment de la sensibilité tactile. Assimilé à un sens 
grossier, le toucher fut considéré comme dangereux sur le plan de la morale, avilissant sur le plan de la sensibilité et source d'erreur sur le plan de la connaissance (Vinit, 2007). Bon dernier dans la classification des sens opéré par la philosophie, le toucher ne fut pas aidé par l'influence de la pensée chrétienne qui le valorisa plutôt sous sa forme négative, dans l'expérience de la privation ou de la souffrance (Classen, 1997).

En dépit du dénigrement qu'il suscite dans la réflexion occidentale, le toucher reste pourtant, sur le plan concret, la première expérience sensorielle de l'être humain et l'une des plus fondamentales à sa survie physique et psychique. La théorie analytique, si elle a encadré l'usage du toucher au sein de la cure pour des raisons liées à l'éthique et à l'interprétation du transfert, a parallèlement souligné, dans son élaboration des différentes étapes de maturation du psychisme, l'importance de la peau dans les échanges précoces du petit enfant.

Abrité dans le giron maternel, l'enfant à naître construit lentement un corpsenveloppe qui se déploie autour et à partir du noyau originaire. La peau constitue ainsi le premier organe du corps (par ailleurs le plus important en taille) et celui qui permet chez le foetus en formation les débuts d'une communication sensorielle. Baigné dans le liquide amniotique, le bébé est en effet capable de ressentir dès la dixième semaine de gestation les nuances très fines de nombreuses stimulations (pressions, bercements, vibrations, mouvements de la main de la mère sur son ventre) :

«Dès les sensations maternelles, ce sont les sensations cénesthésiques qui font du volume du corps résistance à l'invasion du flot amniotique et donnent au corps sa première limite. Les premiers signifiants portés par la voix sont liés aux sensations cénesthésiques de tension et de limite, aux éléments vestibulaires qui permettent de repérer sa position dans l'espace, avec le tact et la peau. » (Clerget, 1997, 47)

Au fur et à mesure de sa croissance, le bébé est serré contre la paroi utérine, exposé aux frottements de son corps à chacun de ses mouvements dans le liquide environnant. Remplaçant le léchage que l'animal reçoit à sa sortie du ventre de sa mère, les contractions utérines de l'accouchement poursuivront chez le petit humain cette stimulation de l'enveloppe corporelle ainsi que l'éveil du système nerveux du bébé.

À la naissance, confronté à la pesanteur de l'air, le nourrisson perd la fluidité légère de ses mouvements in utero. Son corps connaît désormais le poids de la masse corporelle et l'impuissance à bouger librement. Cette incapacité à mouvoir son corps et à se nourrir sans une aide extérieure rend sa survie absolument dépendante de son environnement. À ce stade de son développement psychique, le bébé naissant n'a pas la conscience de son unité corporelle ni des limites créées par sa peau. Cet état de «désintégration» décrit par Winnicott (1971) est à 
l'origine des angoisses archaïques du nourrisson, angoisse de chute, de vide et de liquéfaction que la présence attentive de la mère permettra de contenir.

Dans les soins quotidiens, l'enfant n'est en effet pas seulement en contact avec le sein de sa mère, avec le lait tété ou le tissu du linge qu'on change, mais également avec la manière dont ces gestes sont faits et dont ils s'imprègnent en lui. Avec le regard de sa mère, avec la gamme de sentiments, de pensées (conscientes ou inconscientes) ou de fantasmes qui l'habitent et lui donnent une certaine attitude corporelle. De même, la langue parlée par les parents prend pour l'enfant naissant l'aspect d'un visage, d'une expression, d'une odeur ou d'un bruissement de sons, le sens des mots s'inscrivant d'abord dans une sensation du corps. La peau occupe donc une place fondamentale dans la relation de confiance progressivement tissée par l'enfant avec son environnement. La justesse de l'écoute des besoins du bébé, l'adéquation entre les soins donnés et l'engagement affectif de celui qui les donne «accorderont» progressivement l'enfant à son propre corps.

La psychanalyste Esther Bick dans son travail d'observation des nourrissons souligne à cet égard l'importance des premiers échanges entre la mère et son enfant. Le corps à corps avec la mère crée une peau commune, véritable «digue» corporelle et psychique aux angoisses de l'enfant et ce qu'elles comportent comme risques de dépersonnalisation. C'est cet état de fusion avec la mère qui autorise chez l'enfant la création de sa propre peau psychique:

«Dans sa forme la plus primitive, la personnalité est constituée de parties vécues comme n'ayant pas de liens de cohésion intrinsèque et qui doivent donc être tenues ensemble d'une manière qui est vécue comme passive par la peau qui sert de frontière. Mais cette capacité interne de contenir les parties du self dépend de l'introjection d'un objet externe vécu comme apte à satisfaire ce rôle. » (Bick, 1968)

La mère sert ainsi littéralement de seconde peau à son enfant, recréant dans sa manière de prendre soin de lui, le giron sécurisant offert pendant la grossesse. C'est à travers cette proximité physique et affective que l'enfant va intégrer dans son propre corps les notions de spatialité (la peau touchée délimite l'espace en deux éléments, intérieur et extérieur) et de continuité (par le développement de la sensation d'unité du corps): la capacité contenante remplie par la peau physique lui donne progressivement un sentiment d'existence individuelle suffisamment sécurisante. Plus tard, entre trois et six mois, l'enfant développe sa capacité sensorielle et ses liens avec l'environnement, ce qui entraîne un déplacement des investissements internes jusqu'à la peau et les organes périphériques. Le Moi de l'enfant naît ainsi de la transposition sur le plan inconscient des différentes fonctions de la peau (fonction de maintenance, contenance, pare-excitation, individuation, intersensorialité, soutien de l'excitation sensorielle, recharge libidinale, inscription de traces sensorielles) (Anzieu, 1995, 61): 
«Le Moi-peau acquiert le sentiment de sa continuité temporelle dans la mesure où le Moi-peau se constitue comme une enveloppe suffisamment souple aux interactions de l'entourage et suffisamment contenante de ce qui devient alors des contenus psychiques.» (Anzieu, 1995, 65)

Le toucher de la peau du bébé comme le contact affectif qu'il transmet apparaissent donc comme des éléments fondamentaux de la survie physique du bébé autant que de son développement psychique harmonieux. (Esther Bick illustre les signes indiquant que la mère n'a pas été disponible pour permettre cet état d'unification intérieure du bébé: celui-ci tente ainsi de se «tenir ensemble» à travers de multiples mouvements des membres, en s'accrochant de manière fixe à un stimulus sensoriel extérieur (par exemple au regard) ou encore à travers une tension musculaire faisant office de cuirasse musculaire (Bick, 1968, Dohmen 1992).

Le fait d'être ainsi reconnu (et contenu) dans son immaturité psychique autant que dans sa propre singularité d'être humain (qu'on invite à la rencontre) s'amorce dès les premiers moments du développement de l'enfant. Les neuf mois de la vie utérine participent donc de cet ancrage d'un sentiment de sécurité et de confirmation affective du bébé naissant.

\section{Une pratique singulière autour du toucher : l'haptonomie.}

«La lumière écarte doucement l'obscurité de la chambre. J'ouvre les yeux. Mon corps est calme et reposé. Je sens Maria endormie au-dedans de moi, sage dans le silence aquatique. Je m'étire longuement. Soudain je prends peur, j'ai besoin de la sentir, de la rappeler au monde des vivants. J'approche ma main du ventre rond. J'appelle l'enfant de ma présence. Je suis toute entière dans ma paume offerte au creux de la peau. Maria s'avance, elle semble caresser ma main, s'amuser à venir à son contact. Je bouge mon bras, dessinant un trajet sur les bords du ventre. Maria joue, culbute d'un lieu à l'autre. Je ris de la sentir si vivante au-dedans de moi.» (Vinit, $2004^{1}$ ).

Cette courte vignette illustre la particularité du lien développée par la femme enceinte avec un être humain qu'elle enveloppe et qui pourtant se distingue d'elle par la capacité qu'il démontre de répondre ou de refuser les invitations au contact. L'haptonomie prend appui sur ce geste immémorial des femmes touchant leur ventre pour contacter leur petit enfant. S'appuyant sur la notion de corporéité développée par Merleau Ponty (1945), elle s'est attachée à penser et décrire une phénoménologie du contact affectif. Le corps mis en œuvre n'est ici pas l'objet décrit par la science anatomique mais celui qui crée, en tant que corps animé ouvert à la relation à l'autre, un espace de rencontre. 
Plus concrètement, ce que Veldman nomme la «perception haptique», ce prolongement mis en œuvre pour entrer en relation avec l'enfant à naître, s'illustre dans sa forme la plus commune dans les gestes les plus simples du quotidien: sentir que le caramel au fond de notre casserole n'est pas en train d'attacher, sentir le sol à travers la serpillère ou même installer une vis dans un mur et en appréhender la dureté. Elle constitue une forme de prolongement de notre corporalité aux objets utilisés. La perception haptique dans l'espace est une autre forme de cette capacité de prolongement. Elle a lieu lorsque le danseur sent l'espace qui le sépare de son partenaire de danse ou du fond de la scène et y ajuste son mouvement. Particulièrement affiné chez les artistes corporels elle est aussi utilisée spontanément lorsque nous stationnons notre automobile en intégrant son volume dans notre espace corporel (Revardel, 2007).

Lorsqu'à cette perception haptique s'ajoute une dimension affective, le type de contact mis en oeuvre permet de rejoindre l'autre dans sa corporalité, c'est à dire dans l'être au monde singulier que son corps ouvre dans l'espace:

«Le concept d'haptonomie provient de la conjonction du terme grec classique (hapsis) qui signifie le toucher, le sens, la sensation, le tact, et du nomos qui veut dire règle, loi, norme. Hapto du verbe haptein signifie: je touche, je réunis, j'établis une relation, je m'attache à et au sens figuré: j'établis tactilement un contact pour rendre sain, pour guérir (rendre entier) pour confirmer (l'autre dans son existence).» (Veldman, 1989, 36)

Durant la grossesse, en favorisant la communication entre le bébé et ses parents, l'haptonomie s'attache à redonner une place à l'expérience sensorielle et affective des parents. En se rapprochant de la surface épidermique, en venant se loger sous la main qui l'appelle ou en jouant avec elle, le bébé répond à la sollicitation qui lui est faite. Le couple développe une qualité de présence à l'enfant: les mères apprennent à sentir « de l'intérieur» où est leur enfant (sans systématiquement avoir recours à la palpation de leur ventre, réflexe que l'on retrouve souvent aux débuts des séances); les pères entrent en relation avec l'enfant de manière plus concrète qu'ils n'ont pu le faire jusqu'alors (s'ils n'avaient pas investi la possibilité d'un échange personnel avec l'enfant) ou développent et confirment leur capacité à inviter et répondre au bébé.

Par la place donnée à l'expérience subjective, l'haptonomie constitue également une forme de résistance à la médicalisation de la grossesse et un retour à «l'éprouvé» corporel comme guide de l'intervention obstétrique. Catherine Dolto Tolitch, pédiatre formée à l'haptonomie explique ainsi tout le mouvement qu'il est possible de sentir dans un corps animé, bien à distance des descriptions anatomiques fondées sur le paradigme du corps cadavre:

«Les trompes de Fallope, souples et ondulantes, le souffle de la mère, les vaisseaux sanguins qui se dilatent ou se contractent, les 
parois utérines qui passent de la souplesse accueillante à la rigidité hostile, le péristaltisme digestif de la mère sans cesse en action. Sans oublier les articulations qui modifient la place dans l'habitacle, et les vibrations des voix (de la mère et du père) qui font courir des ondes sur la peau de l'enfant dans le giron. Plus secrètement, il y a les mouvements des cellules qui se multiplient, migrent, se différencient. Enfin les mouvements d'âme de la mère en fonction de sa relation au père et à l'enfant qui se traduisent immédiatement par des modifications des tonus et des rythmes cardiaque et respiratoire. » (Dolto Tolitch, 2008)

L'accord entre les diaphragmes pelviens et thoraciques qui se mettent à bouger comme un tout lorsque la future maman porte affectivement son enfant dans sa base donne tout son sens au mot de giron (souvent utilisé en haptonomie) pour faire apparaître le ventre dans toute la plénitude et l'habitation de cet accueil du bébé, un ventre berceau.

Rappelons pour finir que les rencontres haptonomiques ont toujours lieu en présence de la mère et du père (ou de son représentant) et ne se réduisent pas à une proposition d'exercices, une méthode de stimulation du bébé ou de recherche de performance. L'atmosphère de la rencontre pour reprendre le terme de Tellenbach (1985) est de nature affective : il s'agit d'un dialogue passant par le biais de la peau avec un bébé qui est respecté dans ses rythmes et dans sa disponibilité. Si tout un marché de l'accompagnement prénatal se développe aujourd'hui, Veldman s'est toujours fermement opposé à ce que l'haptonomie soit appris comme une «technique» parmi d'autres, soulignant au contraire combien elle demande un long cheminement personnel pour développer sa propre sécurité de base et qualité d'ouverture à la relation. (Veldman, 1989, 35).

\section{Exemples cliniques}

Dans d'autres cultures, l'enveloppement du bébé dans le giron de la mère se prolonge à travers un portage durant plus d'une année; à l'inverse les enfants occidentaux sont souvent privés rapidement d'un contact rapproché suffisamment sécurisant. La possibilité d'une confirmation affective commençant dès la vie utérine aurait sans doute une incidence sur la sécurisation de l'enfant et le développement confiant de sa relation à l'autre. Nous distinguerons plusieurs niveaux d'intérêt pour une telle pratiqueé

\section{Au niveau du développement psychique du foetus}

En étant invité et reconnu comme un être singulier, le bébé à naître instaure in utéro des échanges signifiants avec ses parents. Au vu des réflexions de D. Anzieu et $\mathrm{E}$. Bick évoqués précédemment, le développement de ce dialogue précoce pourrait contribuer à l'inscription corporelle d'une sécurité de base que des échanges «suffisamment bons» avec l'entourage après la naissance viendront 
prolonger. Sans faire de conjecture sur un vécu fœtal encore peu connu, nous pouvons néanmoins constater la tendance des parents ayant suivi un accompagnement haptonomique à poursuivre après la naissance, une qualité d'écoute des besoins de l'enfant naissant (Dolto Tolitch, 2008).

\section{Au niveau du processus d'attachement mère-enfant}

La maternalité désigne pour Racamier l'intégration pendant l'expérience de la maternité de l'ensemble des processus psychoaffectifs permettant à la femme enceinte de devenir pleinement mère et notamment de faire le deuil de l'enfant imaginaire fantasmé durant la grossesse pour investir son enfant de chair et de sang (Racamier, 1963). Le fait de sentir dans sa corporalité l'enfant à naître et de pouvoir développer avec lui un contact complice pourrait s'inscrire dans une prévention des troubles d'attachement en ancrant la relation mère-enfant dans l'expérience d'un échange affectif commencé dès les débuts de la grossesse. Dolto Tolitch (1977) mentionne la diminution des troubles psychiques du post partum lors d'accompagnements haptonomiques : d'une part en permettant aux femmes ayant accouché de retrouver la sensation de leur propre sécurité de base une fois l'enfant né (de faire le deuil de la fusion autrefois permise avec le bébé), d'autre part en créant un contact affectif signifiant favorisant leur lien d'attachement avec lui.

Annie est enceinte de son deuxième enfant. Très en demande d'un accompagnement haptonomique notre relation se heurte très vite à de multiples empêchements (son téléphone ne fonctionne pas à plusieurs reprises, elle répond très tardivement à mes retours d'appels, rendant très difficile à chaque fois la prise de rendez vous). Lors de la première rencontre avec elle et son conjoint, où je pose le cadre de cette approche, elle ne mentionne rien de particulier au sujet de cette grossesse pleinement désirée. Lors de notre rencontre ultérieure, après avoir pris contact avec son bébé je l'invite doucement dans la partie droite du ventre, ce à quoi ce dernier répond étonnamment vite pour une première expérience de ce type: un bébé déjà bien avancé dans sa croissance utérine (28 semaines). Annie mentionne plusieurs fois qu'elle est très étonnée de cette réaction du bébé, qui n'a jamais bougé ainsi. Elle est prise d'une grande émotion lorsque les mouvements de l'enfant se confirment avec son conjoint.

J'observe la tendance claire du bébé à revenir dans la partie droite du ventre, à s'y lover comme s'il ne voulait plus la quitter. La mère mentionne cette même perception en répétant à nouveau que le bébé n'est jamais allé dans cette zone là. Elle exprime sentir son ventre «équilibré à l'intérieur d'elle». A la fin de la séance lorsque nous faisons un retour sur ce qui a eu lieu durant la séance, elle me confie alors avoir perdu un des bébés dans les toutes premières semaines de la grossesse, chose qu'elle n'avait jamais mentionné auparavant. Par cette invitation à habiter l'ensemble du corps désormais laissé libre par la mort de son jumeau le bébé est concrètement invité à prendre toute la place qui lui revient désormais. Pour la mère, la sensation d'un ventre habité dans tout son espace, permet d'ouvrir la 
thématique du deuil de l'enfant mort ainsi que l'ambivalence d'accueillir en même temps la manifestation de vie d'un autre bébé pleinement présent.

Un deuxième exemple illustrera la pertinence d'un accompagnement haptonomique lors d'une grossesse venue rapidement après une précédente.

Sonia est enceinte de son second bébé, arrivé par surprise 10 mois après la naissance du premier. Cette nouvelle grossesse arrive dans une période déjà très remplie par les soins requis par le premier bébé auquel s'ajoute la reprise d'études universitaires du nouveau papa. Sonia arrive à la première séance avec son conjoint très avancée dans sa grossesse (30 semaines) se sentant coupable de ne pas s'être occupée de ce bébé là comparativement au premier. Elle pleure presque immédiatement lorsque je l'invite à prendre contact par ses mains avec son bébé, en étant attentive à sa sensation interne. Elle se demande alors si elle aime réellement ce bébé qu'elle dit ne pratiquement jamais ressentir malgré l'avancée de la grossesse («il a l'air petit, coincé au fond du ventre, comme s'il se méfiait de moi » me dit-elle).

Lors de cette séance, le père entre rapidement en contact avec un bébé qui semble avide de contacts et répond aux invitations par de longs mouvements doux. La mère continue à dire qu'elle ne ressent pas l'enfant, se dit déçue de la séance et évoque même le fait que son bébé la punit de son manque de présence depuis le début de la grossesse.

Pourtant, lors de la rencontre suivante Sonia arrive en me disant que cette fois elle a senti son bébé durant le temps espaçant nos deux rencontres. Elle a dégagé un moment, juste avant le repas, où elle et son conjoint jouent à dire «bonjour au bébé ». Ce moment consacré à la rencontre de cet enfant à naître se traduit par le sentiment «d'être plus proche de lui et l'impression qu'il ne lui en veut pas. » Elle exprime le fait qu'elle a pu lui parler, expliquer qu'elle était très occupée par son petit frère mais qu'elle souhaite pourtant sa venue. Le fait de sentir dans son corps un espace d'accueil, de faire du «oui» au bébé une ouverture concrète dans son bassin semble avoir créé un espace d'intimité permettant la rencontre avec le bébé et parallèlement le déploiement de son ventre-giron qui cessa désormais de se contracter (Sonia avait des contractions régulières qui faisaient craindre un accouchement prématuré). Le fait que le père entre en relation avec l'enfant contribua sans doute également à permettre à Sonia de faire de la place en son sein pour accueillir ce second bébé.

Dans un autre contexte où les enjeux d'attachement sont particulièrement sollicités, citons l'exemple de mères toxicomanes coupées par l'addiction de leur relation à leur propre corps transformé par la grossesse: Jean Ebert témoigne combien le fait de sentir leur ventre habité par l'enfant peut amener chez des mamans héroïnomanes un nouvel investissement de la grossesse, comme si le ventre s'intégrait, à travers l'expérience affective, à leur image du corps (Ébert, 
2001): «L'évolution de la grossesse a été plusieurs fois modifiée dans le sens d'une maturation de la relation mère-enfant avec comme pour conséquence première une meilleure conscience de soi, une plus grande attention à soi et par conséquence au développement du sentiment de responsabilité de l'enfant dans son giron.»

\section{Au niveau de la place du père}

Le fait de pouvoir développer des relations avec le bébé permet au père de prendre sa place de tiers dans la dyade mère-enfant et de s'engager affectivement dans la grossesse. Dans ma propre pratique, je constate que les femmes ayant un compagnon ayant un métier très prenant ou soumis à des contraintes importantes d'horaires font des séances l'occasion d'une réunion du couple autour de l'enfant. Si la séance peut constituer le premier lien tissé entre le père et l'enfant il importe que la poursuite de ce contact soit la responsabilité du couple. En aucun cas le praticien en haptonomie ne s'y substitue. Inversement certains pères arrivent très en demande de développer un lien avec leur enfant et le rencontrent après la naissance en mentionnant le «connaître déjà ».

\section{Au niveau social}

L'haptonomie trouve enfin un intérêt plus contemporain face à la médicalisation croissante de la grossesse. L'échographie, si elle peut être un moment de joie soutenant l'investissement de la grossesse (à la fois sur le plan de l'imaginaire et du réel), s'avère très souvent une étape de stress intense pour les parents: d'une part en raison de sa réduction trop fréquente à un geste technique, sans présence affective, d'autre part en raison de la charge émotionnelle de l'examen. Le réel de l'image n'est ici pas un support à la rêverie et à l'investissement du bébé mais un verdict de sa normalité et très souvent de sa viabilité.

Marie est enceinte pour la deuxième fois. Lors de sa première grossesse, après toute l'émotion de ce qu'elle pensait être son bébé, elle apprend lors de la seconde échographie qu'elle porte un oeuf clair dont il lui faudra avorter artificiellement à la douzième semaine de gestation. Enceinte à nouveau elle arrive à la rencontre d'haptonomie car on lui a parlé de cette approche pour «être rassurée et sentir le bébé.» Pourtant elle reste très à distance de l'expérience de présence au bébé, s'offrant comme un ventre passif au toucher des mains de son conjoint et au mien. Devant mon invitation à prendre contact avec l'enfant avec ses propres mains placées sur son ventre, puis au dessus de celles de son mari, elle me dit avoir peur de s'attacher à cet enfant là pour risquer finalement qu'il ne meure.

L'expression de cette angoisse permet de s'ouvrir à ce nouveau bébé et d'apprivoiser le fait qu'il soit pleinement vivant. Elle exprimera lors de la séance ultérieure sa joie et sa timidité à entrer en relation avec l'enfant. Le thème de son interruption de grossesse antérieure revenant à plusieurs reprises dans la suite des rencontres, amènera à la référer pour un travail parallèle d'accompagnement psychothérapeutique avant son accouchement. 
Filigrane, automne 2009

\section{Conclusion}

L'haptonomie ne constitue pas une pratique «spectaculaire». Elle est plutôt l'affinement d'un contact qui existe déjà souvent (et fort heureusement) entre l'enfant et ses parents, contact prenant un sens encore plus important lors de vécus de grossesse marqués par la perte ou la difficulté d'attachement. Il s'agit de faire l'expérience entre le bébé, la mère et le père, d'un contact physique qui soit en même temps un contact affectif, marque de la réciprocité du sens du toucher qui nous rend présent à l'autre tout en étant présent à ce que l'autre sent de nous. L'haptonomie offre un lieu d'expérience où la distance entre la corporéité (en terme anatomique et physiologique) et la compréhension de l'être animé semble se résorber (Revardel, 1999): la mère est guidée dans sa présence à l'enfant, présence déployée dans toute sa corporalité et partagée avec le père. Plus qu'un corps-sac, chose de chair contenue par la frontière épidermique, le toucher fait surgir un corps «affectivement marqué»:

«L'intimité c'est le vécu de l'intériorité, le for intérieur, tout cet espace au creux de nous, si plein de vie, de la vie tout ce que l'on se sent être, toute cette substance qui depuis que l'on est une personne, garde en impression tant de vécus, tant d'émotions, qui avec le temps laissent de douces traces, ou des traits bien acérés et qui ne cesse de se remodeler dans la dynamique de la vie.» (Bolsinger, 2001)

Dans un univers médical où la prise en charge des patients est de plus en plus marquée par la technicisation, la place donnée à l'expérience corporelle et affective ainsi que la réassurance dans ses propres sensations s'avèrent fondamentales pour le bon déroulement de la grossesse et de l'accouchement ainsi que pour le développement d'un sentiment de sécurité propice à l'attachement, autant pour la maman que pour le bébé.

florence vinit département de psychologie université du québec à montréal c.p. 8888 , succ. centre-ville montréal (québec) $\mathrm{h} 3 \mathrm{c} 3 \mathrm{p} 8$

vinit.florence@uqam.ca

\section{Note}

1. Vignette écrite suite aux témoignages obtenus dans le cadre de ma thèse de doctorat: Approche phénoménologique du toucher dans les pratiques soignantes contemporaines, UQAM, 2005. 


\section{Bibliographie}

Anzieu, D., 1985, Le Moi-peau, Paris, Dunod.

Bick, E., 1968, L'expérience de la peau dans les relations précoces, in D. Meltzer et coll., éds, Exploration dans le monde de l'autisme, Paris, Payot, 1975, 240-244.

Buber, M., 1992, Je et Tu, Paris, Aubier-Montaigne.

Bolsigner, L., 2001, L'intimité dans l'accompagnement haptonomique périnatal, Actes du $3^{\mathrm{e}}$ Congrès d'Haptonomie, Présence Haptonomique, 6, 35-49.

Buyjendijk, F. J., 1952, Phénoménologie de la rencontre, Paris, Desclée de Brouwer.

Classen, C., 1997, Foundations for an Anthropology of the senses, International Social Science Journal, 153, 401412.

Clerget, J., 1993, La main de l'autre, le geste, le contact et la peau, approche psychanalytique, in J. Cosnier, M. Grosjean, M. Lacoste, éds, Soins et communications, approches interactionnistes des relations de soins, Lyon, Presses universitaires de Lyon.

Courtecuisse, B., Étude sur la symbolique de la main, un outil essentiel à l'osthéopathie. Thèse soumise pour l'obtention du grade d'ostéopathe. Montréal, Collège d'études ostéopathiques de Montréal.

Dohmen, B., 1992, Le toucher en psychothérapie, alliance de travail et/ou résistance. Tiré à part Psycorps.

Dolto Tolitch, C., 2008, La dépression partagée, clinique haptonomique de la dépression maternelle, Actes du $4^{\mathrm{e}}$ Congrès d'Haptonomie, mars 2008.

Dolto Tolitch, C., 1991, Haptonomie pré et postnatale, Journal de pédiatrie et de puériculture, 1, 36-46.

Ebert, J., 2001, Toxicomanie et grossesse : approche haptopsychothérapeutique, Actes du $3^{\mathrm{e}}$ Congrès d'Haptonomie, mars, 51-59.

Fedida, P., 1976, Le corps dans la relation psychothérapeutique et médicale, in Revue de médecine psychosomatique et de psychologie médicale, 18, 3, 237-260.

Freud, S., 1923, Le moi et le ça, in Essais de psychanalyse, Paris, Payot, 1981.

Haag, G., 1991, De la sensorialité aux ébauches de pensée chez les enfants autistes, Revue internationale de psychopathologie, 3, 51-63.

Howes, D., 2005, Skinscapes: Embodiment, Culture, and Environment, in C. Classen, éd., The Book of Touch, Oxford, Berg Publishers.

Leboyer, F., 1977, Shantala: un art royal traditionnel: le massage des enfants, Paris, Seuil.

Le Breton, D., 2002, Conduites à risque. Des jeux de mort au jeu de vivre, Paris, PUF.

Le Breton, D., 2000, Passions du risque, Paris, Métailié.

Le Breton, D., 2002, Signes d'identité. Tatouage, piercings, et autres marques corporelles, Paris, Métailié.

Merleau Ponty, M., 1945, Phénoménologie de la perception, Paris, Gallimard.

Montagu, A., 1979, La peau et le toucher, Paris, Seuil.

Prayez, P., 1994, Le toucher en psychothérapie, Marseille, Epi.

Racamier, P.C., 1963, Notes concernant les psychoses de la puerpéralité, in D. Meltzer et coll., éds, Perspectives dans le monde de l'autisme, Paris, Payot, 1975, 240-244.

Revardel, J.L., 1999, Considérations épistémologiques à propos de l'haptonomie, Présence Haptonomique, avril, 107127.

Revarder, J. L., 2007, Comprendre l'haptonomie, Paris, PUF.

Veldman, F., 1989, L'haptonomie, science de l'affectivité, Paris, Payot.

Vinit, F., 2007, Le toucher qui guérit, entre soin et communication, Paris, Belin. 
Filigrane, automne 2009

Tellenbach, H., (1985), Goût et Atmosphère, Paris, PUF.

Winnicott, D. W. 1971, Le corps et le self, Nouvelle Revue de psychanalyse, 3, 37-48. 